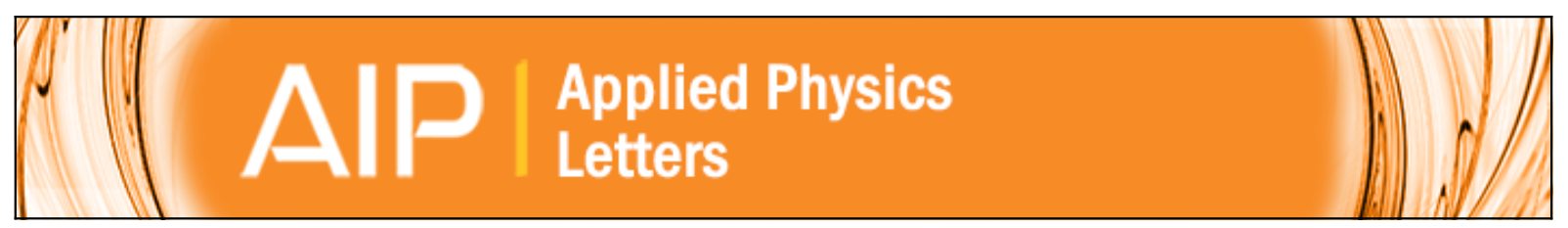

\title{
Spin-dependent terahertz oscillator based on hybrid graphene superlattices
}

E. Díaz, K. Miralles, F. Domínguez-Adame, and C. Gaul

Citation: Applied Physics Letters 105, 103109 (2014); doi: 10.1063/1.4895673

View online: http://dx.doi.org/10.1063/1.4895673

View Table of Contents: http://scitation.aip.org/content/aip/journal/apl/105/10?ver=pdfcov

Published by the AIP Publishing

\section{Articles you may be interested in}

Spin-dependent Seebeck effects in a graphene nanoribbon coupled to two square lattice ferromagnetic leads J. Appl. Phys. 117, 104305 (2015); 10.1063/1.4914486

Spin echo dynamics under an applied drift field in graphene nanoribbon superlattices

Appl. Phys. Lett. 103, 233112 (2013); 10.1063/1.4836395

Spin transport and magnetoresistance in Thue-Morse graphene superlattice with two ferromagnetic graphene electrodes

J. Appl. Phys. 114, 163715 (2013); 10.1063/1.4827380

Electronic and magnetic properties of oxygen patterned graphene superlattice

J. Appl. Phys. 112, 114332 (2012); 10.1063/1.4769743

Graphene-diamond hybrid structure as spin-polarized conducting wire with thermally efficient heat sinks Appl. Phys. Lett. 100, 233101 (2012); 10.1063/1.4725485

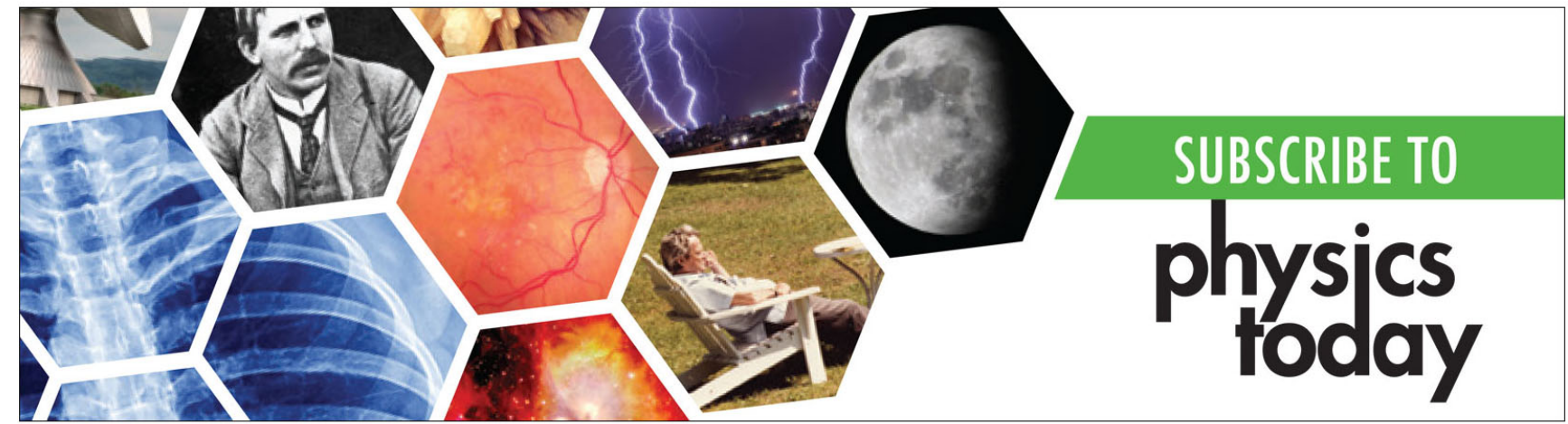




\title{
Spin-dependent terahertz oscillator based on hybrid graphene superlattices
}

\author{
E. Díaz, ${ }^{1}$ K. Miralles, ${ }^{1}$ F. Domínguez-Adame,${ }^{1}$ and C. Gaul ${ }^{2, a)}$ \\ ${ }^{1}$ GISC, Departamento Física de Materiales, Universidad Complutense, E-28040 Madrid, Spain \\ ${ }^{2}$ Max Planck Institute for the Physics of Complex Systems, 01187 Dresden, Germany
}

(Received 3 July 2014; accepted 30 August 2014; published online 12 September 2014)

\begin{abstract}
We theoretically study the occurrence of Bloch oscillations in biased hybrid graphene systems with spin-dependent superlattices. The spin-dependent potential is realized by a set of ferromagnetic insulator strips deposited on top of a gapped graphene nanoribbon, which induce a proximity exchange splitting of the electronic states in the graphene monolayer. We numerically solve the Dirac equation and study Bloch oscillations in the lowest conduction band of the spin-dependent superlattice. While the Bloch frequency is the same for both spins, we find the Bloch amplitude to be spin dependent. This difference results in a spin-polarized ac electric current in the $\mathrm{THz}$ range. (C) 2014 AIP Publishing LLC. [http://dx.doi.org/10.1063/1.4895673]
\end{abstract}

According to the theoretical work by Esaki and Tsu, ${ }^{1}$ negative differential resistance in biased superlattices (SLs) signals the occurrence of Bloch oscillations (BOs). ${ }^{2,3}$ One of the main obstacles for the realization of an active Bloch oscillator is the instability of the electric field, which results in the formation of electric domains. Savvidis et al. found that these domains appear to be suppressed in a InAs/AlSb super-SL composed of many very short segments of SL, interrupted by heavily doped InAs regions. ${ }^{4}$ The stabilization of the electric field in semiconductor SLs can also be achieved by application of the cleaved-edge overgrowth technique. ${ }^{5}$ In any way, the suppression of the electric domains in semiconductor SLs requires fairly complex designs.

Graphene SLs may easily overcome the instability of the electric field since the back gate voltage induces a uniform population of the quantum wells. In addition, the carrier density can be varied over a wide range. Patterning graphene at the nanometer scale can be achieved by hydrocarbon lithography, ${ }^{6}$ chemical functionalization, ${ }^{7}$ or He ion lithography, ${ }^{8}$ which opens a possibility to fabricate these SLs. Graphene SLs have recently been a focus of interest to study a variety of quantum phenomena. $^{9-12}$ Dragoman and Dragoman proposed a SL obtained by patterning an array of metallic electrodes on gapless graphene, where BOs of up to tens of terahertz can be generated due to the low scattering rate in graphene. ${ }^{13}$ In their design, the metallic electrodes are inclined with respect to the current flow to minimize Klein tunneling. Negative differential resistance and the WannierStark ladder regime in semiconducting armchair graphene nanoribbon (GNR) SLs has been investigated by Ferreira et al. ${ }^{14}$ When the gap of the GNR is small, besides conventional BOs, multiple Zener tunneling between the coupled electron and hole branches leads to distinct coherent oscillations. $^{15}$

Spin-related and magnetic effects are of special interest for their relevance in spintronics. ${ }^{16-20}$ Recently, we proposed a hybrid SL realized by $\mathrm{EuO}$ ferromagnetic insulator strips deposited on top of a GNR. ${ }^{21}$ These strips induce a proximity exchange splitting of the electronic states in graphene, ${ }^{22}$

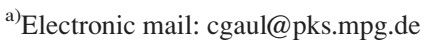

resulting in the appearance of a SL with a spin-dependent potential profile. The electric current through the hybrid SL can be highly polarized and manifests spin-dependent negative differential resistance. ${ }^{21}$

In this paper, we investigate the high-frequency dynamics of electrons in a hybrid SL formed by a periodic arrangement of ferromagnetic strips grown on top of an armchair GNR. As mentioned above, the ferromagnetic strips induce a spin-dependent potential. Therefore, we expect spindependent BOs when the hybrid SL is subjected to a voltage drop between the source and the drain. We find that the Bloch frequency agrees with the semiclassical prediction and that the Bloch amplitude does so for sufficiently wide wave packets. Interestingly, as it occurs in the case of Bloch oscillators based on semiconductors, the present design also generates electric currents in the $\mathrm{THz}$ range. However, the amplitude of the BOs are spin dependent, and consequently the generated ac electric current is spin polarized.

The hybrid system consists of a rectangular GNR of width $W$, connected to source and drain leads, on top of which there are $N$ ferromagnetic insulator strips of width $w_{a}$, with the spacing between them being $w_{b}$ [see Fig. 1(a)]. We restrict ourselves to GNRs with armchair edges hereafter. Experimental evidences ${ }^{23}$ and ab-initio calculations ${ }^{24}$ show that the energy spectrum presents a gap inversely proportional to the width $W$, depending on the remainder $\left(2 W / a_{0} \bmod 3\right)$, where $a_{0}=0.246 \mathrm{~nm}$ is the lattice constant, namely, the width of the graphene lattice hexagon. Contrary to GNRs with zigzag edges, the dispersion relation of the armchair ones is centered around $k=0$, making the resonant levels broader and less affected by disorder. ${ }^{25}$

The proximity exchange interaction between magnetic ions in the strips and charge carriers in the GNR can be described as an effective Zeeman splitting $\pm \Delta_{\mathrm{ex}}$ of the spin sublevels. ${ }^{22}$ We use $\Delta_{\mathrm{ex}}=8 \mathrm{meV}$ as a typical value; we have checked that our results do not change qualitatively if we use a different value of $\Delta_{\mathrm{ex}}$ within the range of a few $\mathrm{meV}$. The proximity exchange interaction has the characteristic length scale of one atomic layer. Therefore, the splitting is induced only in the regions of the GNR directly below the ferromagnetic strips. Consequently, a spin-up (spin-down) electron propagating along the sample will be subjected to a potential 


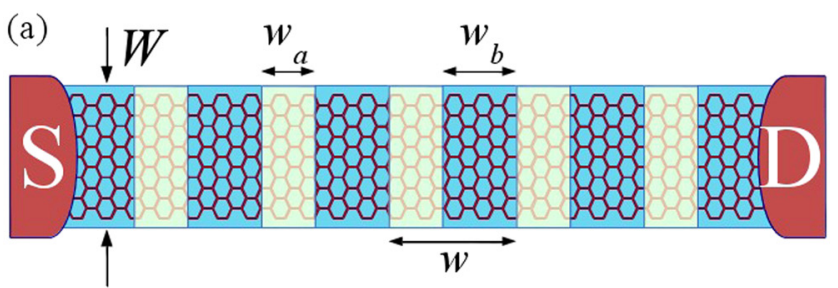

(b)

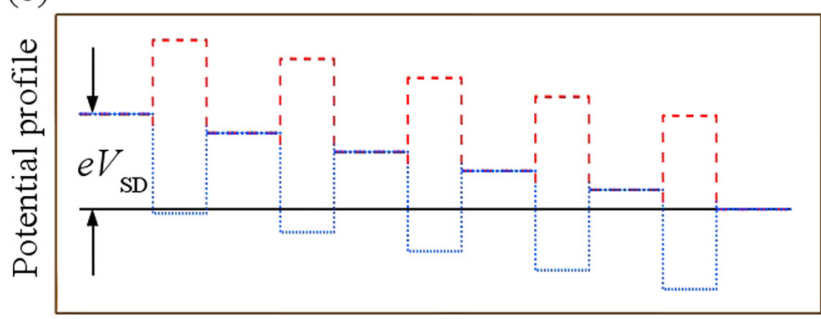

comprising a periodic set of rectangular barriers (wells), as plotted in Fig. 1(b).

For not too narrow GNRs, the low energy excitations can be treated very efficiently within the Dirac approximation. ${ }^{26}$ The wave function on each sublattice $\alpha=a, b$ is expanded around both Dirac points $\boldsymbol{K}$ and $\boldsymbol{K}^{\prime}=-\boldsymbol{K}$, which are also referred to as valleys

$$
\phi_{\alpha}(\boldsymbol{r})=e^{\mathrm{i} \boldsymbol{K} \cdot \boldsymbol{r}} \psi_{\alpha}^{+}(\boldsymbol{r})+e^{-\mathrm{i} \boldsymbol{K} \cdot \boldsymbol{r}} \psi_{\alpha}^{-}(\boldsymbol{r}) .
$$

In coordinates where $\boldsymbol{K}$ points lie in the $y$ direction [see Fig. 1(d)], the Dirac equation reads

$$
\mathrm{i} \hbar \frac{\partial}{\partial t}\left(\begin{array}{l}
\psi_{a}^{ \pm} \\
\psi_{b}^{ \pm}
\end{array}\right)=\left(\begin{array}{cc}
\mathcal{V}(x) & v_{\mathrm{F}}\left(-\mathrm{i} \hat{p}_{x} \mp \hat{p}_{y}\right) \\
v_{\mathrm{F}}\left(\mathrm{i} \hat{p}_{x} \mp \hat{p}_{y}\right) & \mathcal{V}(x)
\end{array}\right)\left(\begin{array}{l}
\psi_{a}^{ \pm} \\
\psi_{b}^{ \pm}
\end{array}\right) .
$$

Here, $v_{\mathrm{F}}=3 t_{0} a_{0} / 2=10^{6} \mathrm{~m} / \mathrm{s}$ is the Fermi velocity in graphene, with $t_{0}$ being the nearest-neighbor hopping energy in the honeycomb lattice. In $y$ direction, the boundary conditions require the wave function to vanish on the (fictitious) sites just outside the GNR, i.e., at $y=0$ and $y=W+a_{0}$, where the $y$ axis is perpendicular to the direction of the GNR, whose lower edge is located at $y=a_{0} / 2$ [see Fig. 1(c)]. In the present case of armchair GNRs, boundary conditions are fulfilled by a superposition of two states from different valleys with the same longitudinal wave function but with opposite transverse wave number: $\psi_{\alpha}^{ \pm}(x, y)=\exp \left( \pm \mathrm{i} k_{\perp} y\right) f_{\alpha}(x)$. The possible values of $k_{\perp}$ depend crucially on the width $W$ of the GNR and on $\left(2 W \bmod 3 a_{0}\right) \cdot{ }^{27,28}$ In the gapped cases, which are of interest here, the lowest value is $k_{\perp} \approx \pi / 3 W$. The transverse part of $\phi_{\alpha}$ is a rapidly varying standing wave of the form $\sin \left[\left(K+k_{\perp}\right) y\right]$. The longitudinal wavefunction $f_{\alpha}(x)$, on the contrary, varies smoothly. Its equation of motion is a onedimensional Dirac equation

$$
\mathrm{i} \hbar \frac{\partial}{\partial t}\left(\begin{array}{l}
f_{a} \\
f_{b}
\end{array}\right)=\left(\begin{array}{cc}
\mathcal{V}(x) & \hbar v_{\mathrm{F}}\left(-\partial_{x}-k_{\perp}\right) \\
\hbar v_{\mathrm{F}}\left(\partial_{x}-k_{\perp}\right) & \mathcal{V}(x)
\end{array}\right)\left(\begin{array}{l}
f_{a} \\
f_{b}
\end{array}\right) .
$$

For a constant potential $\mathcal{V}$, the solution of the onedimensional Dirac equation in terms of plane waves is

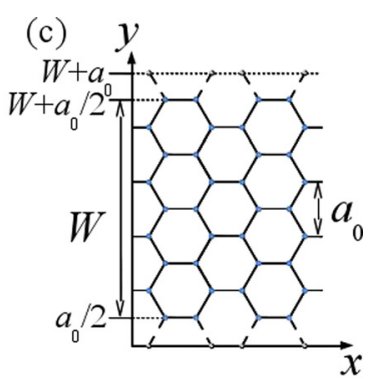

FIG. 1. (a) Sketch of the setup: The GNR is connected to source (S) and drain (D) leads, with $N=5$ strips of a ferromagnetic insulator on top of it. (b) Potential profiles for spin-up (dashed red lines) and spin-down (dotted blue lines) electrons in the biased device. (c) Honeycomb lattice structure and edges of the GNR. (d) Brillouin zone of the honeycomb lattice with the Dirac points $\boldsymbol{K}$ and $\boldsymbol{K}^{\prime}$ (blue) and two states (red) whose superposition fulfills the boundary conditions if $k_{\perp}$ is chosen correctly.

straightforward. For piecewise potentials, the solutions can then be matched together by transfer-matrix techniques. ${ }^{21}$ Due to nonlinear dependence on $E-\mathcal{V}(x)$, however, the analytical treatment is difficult. Here, we pursue a different approach and seek to diagonalize the stationary version of (3).

The Dirac equation (3) couples $f_{\alpha}$ to the first derivative $f_{\bar{\alpha}}^{\prime}$ of the respective other sublattice. Thus, it is convenient to sample $f_{b}$ on points that lie just between the points where $f_{a}$ is sampled and to collect the data in an alternating array $g_{j}$ with $g_{2 n}=f_{a}(2 n d), g_{2 n+1}=f_{b}((2 n+1) d)$ and discretization step $d$. The stationary one-dimensional Dirac equation (3) then becomes a one-dimensional tight-binding equation of motion with alternating hopping energies

$$
\frac{E-\mathcal{V}_{j}}{\hbar v_{\mathrm{F}}} g_{j}=(-1)^{j} \frac{g_{j+1}-g_{j-1}}{2 d}-k_{\perp} \frac{g_{j+1}+g_{j-1}}{2},
$$

where $\mathcal{V}_{j}=\mathcal{V}(j d)$. The last term comes from interpolating the wave function on the opposite sublattice for $f_{b}(2 n)$ $=\left[f_{b}((2 n+1) d)+f_{b}((2 n-1) d)\right] / 2$ and similar for $f_{a}((2 n+1) d)$. For vanishing potential, the spectrum of (4) as a function of the longitudinal momentum $k$ is

$$
E_{ \pm}(k)= \pm \hbar v_{\mathrm{F}}\left[k_{\perp}^{2} \cos ^{2}(k d)+d^{-2} \sin ^{2}(k d)\right]^{1 / 2},
$$

with a gap opened by the transverse momentum $k_{\perp}$. In the limit $d \rightarrow 0$, this goes over to the well-known Dirac dispersion $E_{ \pm}(k)= \pm \hbar v_{\mathrm{F}}\left(k_{\perp}^{2}+k^{2}\right)^{1 / 2}$. Equation (5) holds for low energies. The outer band edges $\pm \hbar v_{\mathrm{F}} / d$ are artifacts of the discretization and have nothing to do with the band edges of the honeycomb lattice.

Figure 2 presents the numerically obtained band structure for the infinite untilted SL. While the central gap is again due to the transverse momentum $k_{\perp}$, the other gaps are due to the SL strength $\Delta_{\mathrm{ex}}$. Interestingly, the widths of the bands $B_{\uparrow}$ and $B_{\downarrow}$ are different (1.53 and $1.26 \mathrm{meV}$ for the chosen parameters, respectively). Since the amplitude of the BOs depends on the bandwidth, this difference will ultimately lead to the generation of spin-dependent ac electric current.

We are now in position to numerically diagonalize the Dirac equation. Keeping in mind the properties of the 


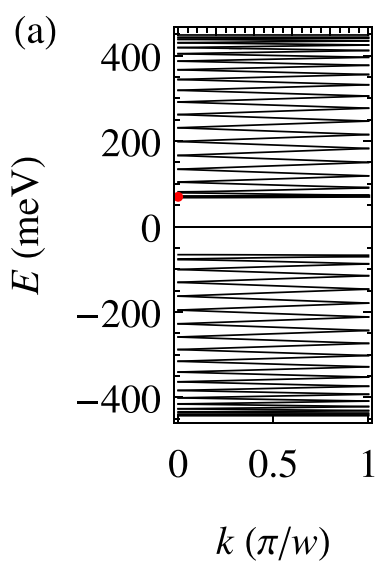

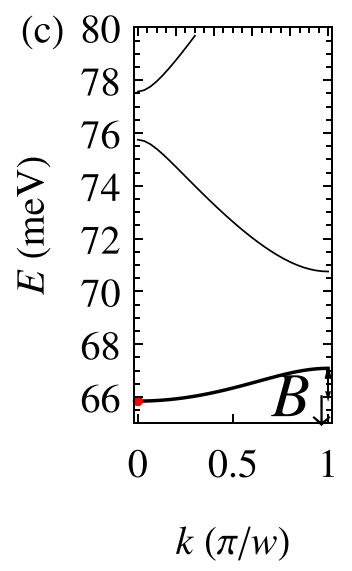

FIG. 2. Band structure from (4) in the case of an infinite untilted SL with $W=9.84 \mathrm{~nm}$, $\Delta_{\mathrm{ex}}=8 \mathrm{meV}, w_{a}=23.9 \mathrm{~nm}$, $w_{b}=83.0 \mathrm{~nm}$, and discretization step $d=w / 72=1.48 \mathrm{~nm}$ with $w=w_{a}+w_{b}$. (a) Full spectrum for a spin-up electron. Panels (b) and (c) show enlarged views of the lowest conduction bands for spin up and down, respectively. untilted SL, $d$ has to be sufficiently small, such that the SL strength $\Delta_{\text {ex }}$ is smaller than the bandwidth (5) to avoid discretization artifacts, namely, $d \ll \hbar v_{\mathrm{F}} / \Delta_{\mathrm{ex}}$. Hereafter, we take $d=w / 72$ with $w=w_{a}+w_{b}$. We have checked that the results are the same within the numerical uncertainty for smaller values of the discretization step. Moreover, in order to have electron and hole states well separated, the SL strength should not exceed the gap of the homogeneous GNR, i.e., $\Delta_{\mathrm{ex}} \ll \hbar v_{\mathrm{F}} k_{\perp}$.

In order to explore BOs in the tilted graphene SL, we consider a system of $N \gg 1$ wells with a source-drain voltage $V_{\mathrm{SD}}$ applied across the whole sample. Then, the energy spectrum of the graphene SL resembles the well-known Wannier-Stark ladder, as shown in Fig. 3. This means that the energy levels $E_{\nu}$ become equally spaced with level spacing $e V_{\mathrm{SD}} / N$ and the eigenstates become localized with a similar envelope function. Only the states at the very edge of the energy spectrum (see, e.g., state labeled $\nu=1$ in Fig. 3) are influenced by finite size effects since they are localized close to the boundaries of the SL. In view of this energy spectrum, BOs are expected to occur in the device.

As initial state we consider the localized wave packet of an electron excited to the lowest conduction band of the SL. Thus, we take the state $k=0$, as marked in Figs. 2(b) and 2(c) by red dots, multiply it with a Gaussian envelope of variance $\sigma^{2}$ centered at $x_{0}$ and normalize the wave function afterwards. In Fig. 4, the initial states for the spin-up and spin-down

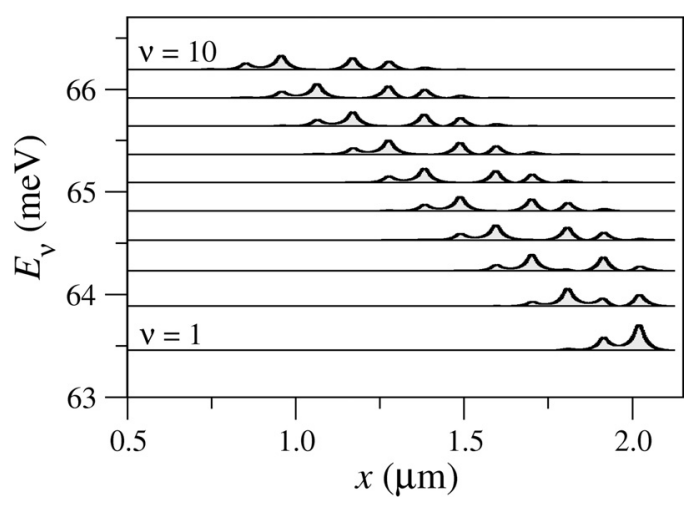

FIG. 3. Probability density of a subset of eigenstates from the lowest conduction band $(\nu=1, \ldots, 10)$ for the spin-down electrons in a graphene SL of $N=20$ wells, $\Delta_{\mathrm{ex}}=8 \mathrm{meV}, e V_{\mathrm{SD}}=5.5 \mathrm{meV}, w_{a}=23.9 \mathrm{~nm}$, and $w_{b}=$ $83.0 \mathrm{~nm}$. The baseline indicates the energy of every eigenstate. The right edge of the plot coincides with the edge of the SL. electrons are shown in a biased lattice of $N=80$ wells. Hereafter, the initial states considered in the numerical simulations will be defined with parameters $\sigma=3 w=320.7 \mathrm{~nm}$ and $x_{0}=3.0 \mu \mathrm{m}$.

The time-dependent wave function is obtained from the expansion of the initial wave function in the eigenstates $g_{j}^{\nu}$ of the tilted system as follows:

$$
g_{j}(t)=\sum_{\nu} c_{\nu} g_{j}^{\nu} e^{-i E_{\nu} t / \hbar}, \quad c_{\nu}=\sum_{j}\left(g_{j}^{\nu}\right)^{*} g_{j}(0) .
$$

We study the electron dynamics by means of the time evolution of the centroid of the wave function

$$
X(t)=d \sum_{j} j\left|g_{j}(t)\right|^{2} .
$$

We also define the dimensionless current $J_{s}(t) \propto\left\langle\sigma_{x}\right\rangle$, where $\sigma_{x}$ is the Pauli matrix and $s$ refers to the spin, as

$$
J_{s}(t)=-\frac{\mathrm{i}}{2} \sum_{i, j} g_{i}(t)^{*}\left(\delta_{i, j+1}-\delta_{i, j-1}\right) g_{j}(t)
$$

The electric current is proportional to the dimensionless current $J_{s}(t)$. In Fig. 5, the time evolution of the centroid for spin-up and spin-down electrons and the net polarized current $J_{\uparrow}-J_{\downarrow}$ in a biased device are shown. Both magnitudes are clearly oscillatory with a well defined frequency of $\omega \approx 0.1 \mathrm{THz}$, which agrees well with the semiclassical estimate of the Bloch frequency $\omega_{\mathrm{B}}=e V_{\mathrm{SD}} / N \hbar$.

Figure 6(a) shows the amplitude $A_{\mathrm{B}}$ of the centroid motion as a function of the inverse of $V_{\mathrm{SD}}$ for both spins and
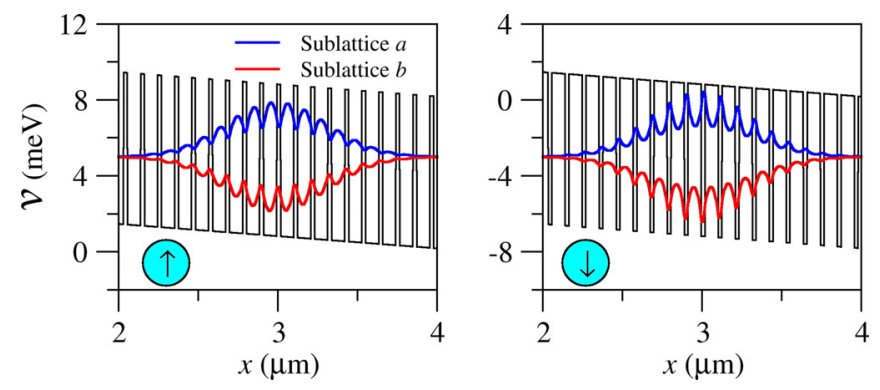

FIG. 4. Potential profile and the initial state (arbitrary units) for spin-up (left) and spin-down (right) electrons in the biased device. Blue and red lines correspond to $f_{a}$ and $f_{b}$, respectively. The parameters are the same as in Fig. 3, but $N=80$ wells, $\sigma=3 w=320.7 \mathrm{~nm}$ and $x_{0}=3.0 \mu \mathrm{m}$. 

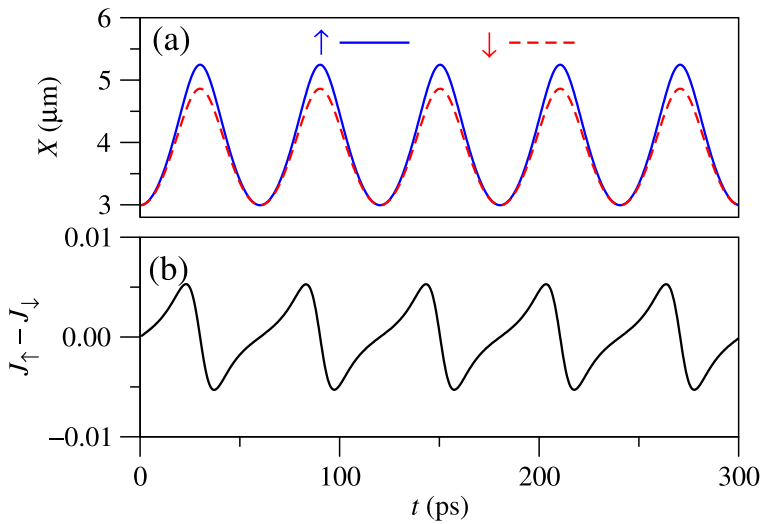

FIG. 5. Time evolution of (a) the centroid of spin-up and spin-down electrons and (b) the net polarized current in the biased device. The current data have been downsampled to a time resolution of 1 ps. Parameters are the same as in Fig. 4.
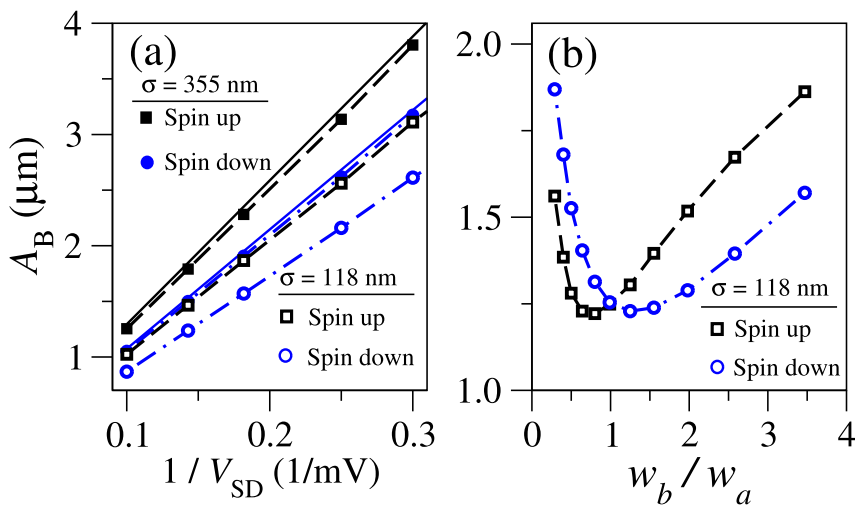

FIG. 6. (a) Bloch amplitude of the spin-up and spin-down electrons as a function of the inverse voltage $1 / V_{\mathrm{SD}}$. Solid lines indicate the semiclassical prediction (infinite-width wave packet). (b) Bloch amplitude as a function of the ratio between the width of the ferromagnetic strips and their spacing, with the constraint $w_{a}+w_{b}=106.9 \mathrm{~nm}$. The other parameters are the same as in Fig. 4.

two different values of $\sigma$. Notice that the wider the wave packet, the better the amplitude approaches the classical estimate $A_{\mathrm{B}}^{\uparrow \downarrow}=B_{\uparrow \downarrow} w / \hbar \omega_{\mathrm{B}}$, where $B_{\uparrow \downarrow}$ is the band width of the lowest conduction band (see Fig. 2). Our simulations recover the expected dependence $A_{\mathrm{B}} \sim \omega_{\mathrm{B}}^{-1} \sim V_{\mathrm{SD}}^{-1}$. More importantly, although electrons with spin up and spin down perform BOs with the same frequency $\omega_{B}$, the amplitude depends on the particular spin state.

In Fig. 6(b), we study the impact of the geometry of the device on the spin selectivity by plotting the amplitude of the BOs as a function of the ratio of the spacing between the strips and the width of the strips. No spin-dependent effect is expected if the widths of both materials are the same. On the contrary if one of the widths is much larger than the other, the difference between the $A_{\mathrm{B}}$ of both spin states is increased. Indeed, it can reach a difference up to $17 \%$ within the considered range of parameters.

In conclusion, we have proposed a new design of $\mathrm{THz}$ oscillator based on hybrid graphene SLs. A spin-dependent potential acts on the electrons due to a set of ferromagnetic insulator strips deposited on top of a GNR. When subjected to a potential drop between source and drain, the electrons excited to the lowest conduction band perform BOs in the $\mathrm{THz}$ range. The frequency of the coherent oscillation is independent of the electron spin. On the contrary, the Bloch amplitude may differ significantly due to the different bandwidths for both spins. The different spatial extent of the electron motion in real space yields a spin-polarized ac electric current in the $\mathrm{THz}$ domain. The resulting ultrafast magnetization could be detected with $\mathrm{THz}$ emission spectroscopy. $^{29}$

We thank E. Diez and Y. M. Meziani for helpful discussions. Work in Madrid was supported by MINECO (Project Nos. MAT2010-17180 and MAT2013-46308).

${ }^{1}$ L. Esaki and R. Tsu, IBM J. Res. Dev. 14, 61 (1970).

${ }^{2}$ F. Bloch, Z. Phys. 52, 555 (1929).

${ }^{3}$ C. Zener, Proc. R. Soc. London, Ser. A 145, 523 (1934).

${ }^{4}$ P. G. Savvidis, B. Kolasa, G. Lee, and S. J. Allen, Phys. Rev. Lett. 92, 196802 (2004).

${ }^{5}$ T. Feil, H.-P. Tranitz, M. Reinwald, and W. Wegscheider, Appl. Phys. Lett. 87, 212112 (2005).

${ }^{6}$ J. C. Meyer, C. O. Girit, M. F. Crommie, and A. Zettl, Appl. Phys. Lett. 92, 123110 (2008).

${ }^{7}$ Z. Sun, C. L. Pint, D. C. Marcano, C. Zhang, J. Yao, G. Ruan, Z. Yan, Y. Zhu, R. H. Hauge, and J. M. Tour, Nat. Commun. 2, 559 (2011).

${ }^{8}$ B. S. Archanjo, B. Fragneaud, L. Gustavo Cançado, D. Winston, F. Miao, C. Alberto Achete, and G. Medeiros-Ribeiro, Appl. Phys. Lett. 104, 193114 (2014).

${ }^{9}$ G. M. Maksimova, E. S. Azarova, A. V. Telezhnikov, and V. A. Burdov, Phys. Rev. B 86, 205422 (2012).

${ }^{10}$ S. Dubey, V. Singh, A. K. Bhat, P. Parikh, S. Grover, R. Sensarma, V. Tripathi, K. Sengupta, and M. M. Deshmukh, Nano Lett. 13, 3990 (2013).

${ }^{11}$ L. A. Ponomarenko, R. V. Gorbachev, G. L. Yu, D. C. Elias, R. Jalil, A. A. Patel, A. Mishchenko, A. S. Mayorov, C. R. Woods, J. R. Wallbank, M. Mucha-Kruczynski, B. A. Piot, M. Potemski, I. V. Grigorieva, K. S. Novoselov, F. Guinea, V. I. Fal'ko, and A. K. Geim, Nature 497, 594 (2013).

${ }^{12}$ F. Sattari and E. Faizabadi, Eur. Phys. J. B 86, 278 (2013).

${ }^{13}$ D. Dragoman and M. Dragoman, Appl. Phys. Lett. 93, 103105 (2008).

${ }^{14}$ G. J. Ferreira, M. N. Leuenberger, D. Loss, and J. C. Egues, Phys. Rev. B 84, 125453 (2011).

${ }^{15}$ V. Krueckl and K. Richter, Phys. Rev. B 85, 115433 (2012).

${ }^{16}$ L. Jiang and Y. Zheng, J. Appl. Phys. 109, 053701 (2011).

${ }^{17}$ Q. H. Huo, R. Z. Wang, and H. Yan, Appl. Phys. Lett. 101, 152404 (2012).

${ }^{18}$ W.-T. Lu, W. Li, Y.-L. Wang, C.-Z. Ye, and H. Jiang, J. Appl. Phys. 112, 083712 (2012).

${ }^{19}$ X.-X. Yu, Y.-E. Xie, Y. T. Ou, and Y.-P. Chen, Chin. Phys. B 21, 107202 (2012).

${ }^{20}$ E. Faizabadi, M. Esmaeilzadeh, and F. Sattari, Eur. Phys. J. B 85, 198 (2012).

${ }^{21}$ J. Munárriz, C. Gaul, A. V. Malyshev, P. A. Orellana, C. A. Müller, and F. Domínguez-Adame, Phys. Rev. B 88, 155423 (2013).

${ }^{22}$ H. Haugen, D. Huertas-Hernando, and A. Brataas, Phys. Rev. B 77, 115406 (2008).

${ }^{23}$ M. Y. Han, B. Özyilmaz, Y. Zhang, and P. Kim, Phys. Rev. Lett. 98, 206805 (2007).

${ }^{24}$ Y.-W. Son, M. L. Cohen, and S. G. Louie, Phys. Rev. Lett. 97, 216803 (2006).

${ }^{25}$ J. Munárriz, F. Domínguez-Adame, and A. V. Malyshev, Nanotechnology 22, 365201 (2011).

${ }^{26}$ A. H. Castro Neto, F. Guinea, N. M. R. Peres, K. S. Novoselov, and A. K. Geim, Rev. Mod. Phys. 81, 109 (2009).

${ }^{27}$ L. Brey and H. A. Fertig, Phys. Rev. B 73, 235411 (2006).

${ }^{28}$ K. Wakabayashi, Y. Takane, M. Yamamoto, and M. Sigrist, New J. Phys. 11, 095016 (2009).

${ }^{29}$ E. Beaurepaire, G. M. Turner, S. M. Harrel, M. C. Beard, J.-Y. Bigot, and C. A. Schmuttenmaer, Appl. Phys. Lett. 84, 3465 (2004). 